\title{
SEVERAL APPROXIMATIONS OF $\pi(x)$
}

\author{
LAURENŢIU PANAITOPOL
}

Abstract. In this paper several new inequalities on the function $\pi(x)$ (numbers of primes not exceeding $x$ ) are presented. In the proofs, essentially the well-known results of Rosser and Schoenfeld are used.

Legendre conjectured that $x /(\log x-A)$ (with $A=1.08366 \ldots$ ) is a good approximation for $\pi(x)$. We prove that, for $x>10^{6}$, the function considered by Legendre is actually an upper bound.

L. Locker-Ernst affirms that $\frac{n}{h(n)}$, with $h(n)=\frac{1}{3}+\frac{1}{4}+\ldots+\frac{1}{n}$ is very close to $\pi(n)$. We precize the above statement by proving that, for $n \geqslant 1429, \frac{n}{h(n)}$ is actually a lower bound for $\pi(n)$.

Mathematics subject classification (1991): 11A25, 11N05.

Key words and phrases: Inequalities for arithmetic functions, bounds for integrals of convex functions, Legendre conjecture.

\section{REFERENCES}

[1] B. P. DEMIDOVICH and I. A. MARON, Elements de calcul numerique, Edition Mir Moscou, p. 594 (1973).

[2] L. LOCKER-ERnSt, Bemerkungen über die Verteilung der Primzahlen, Elemente der Mathematik XIV, nr. 1, 1-5, Basel (1959).

[3] D. S. MitrinOviC and J. SANDOR (in coop. with B. Cristici), Handbook of number theory, Kluwer Acad. Publ., cap. VII (1996).

[4] N. C. PEREIRA, Elementary estimate for the Chebyshev function $\psi(x)$ and the Mobius function $M(x)$, Acta Arith. 52, 307-337 (1989).

[5] P. RibenBoim, The book of prime number records, Springer Verlag, p. 159 (1996).

[6] J. B. Rosser, Explicit bounds for some functions of prime numbers, Amer. J. Math. 63, 211-232 (1941).

[7] J. B. ROSSER and L. SCHOENFELD, Approximate formulas for some functions of prime numbers, Illinois J. Math 6, 64-94, (1962).

[8] J. B. Rosser and L. SCHOENFELD, Sharper bounds for the Chebyshev functions $\theta(x)$ and $\psi(x)$, Mathematics of Computation 29, no. 129, 243-269, (1975).

[9] L. SCHOENFELD, Sharper bounds for the Chebyshev functions $\theta(x)$ and $\Psi(x)$ II, Mathematics of Computation, vol. 30, nr. 134, 337-360, (1976). 